\title{
Neue Beiträge zur Kenntnis des Cholesterins.
}

\author{
I. \\ Über Anlagerung von Chlorwasserstoff \\ von \\ J. Mauthner. \\ (Vorgelegt in der Sitzung am 22. März 1906.)
}

Seit langer Zeit ist es bekannt, daß Cholesterin und seine Ester Halogen addieren, derart, daß eine Doppelbindung im Molekül angenommen werden muß, dagegen sind Produkte der Anlagerung von Halogenwasserstoff bisher nicht beschrieben worden.

Im Jahre 1894 haben W. Suida und $\mathrm{ich}^{1}$ berichtet, daß Cholesterin und Cholesten, mit aikoholischer Salzsäure behandelt, chlorhaltige, kristallinische Körper liefern. Über das damals aus dem Cholesterin erhaltene Produkt konnte nicht gleich Klarheit gewonnen werden, da es keine konstante $\mathrm{Zu}$ sammensetzung zeigte und sich als leicht zersetzlich erwies. Später hielten wir es für nicht unmöglich, daß hier eine salzartige Verbindung vorliege, so wie dies bei dem essigsauren Cholesterin (Hoppe-Seyler) und dem von uns beschriebenen oxalsauren Cholesterin ${ }^{2}$ der Fall ist.

Das vor einiger Zeit wieder aufgenommene Studium der Reaktion führte nun zu dem Ergebnis, daß sich Chlorwasserstoff

1 Mauthner und Suida, Beiträge zur Kenntnis des Cholesterins, II. Abhandlung. Monatshefte für Chemie, XV (1894), p. 374.

2 Mauthner und Suida, Beiträge zur Kenntnis des Cholesterins, VI. Abhandlung. Monatshefte für Chemie, XXIV (1903), p. 664. 
an die doppelte Bindung anlagert; ebensowohl beim Cholesterin selbst wie beim Cholesterylchlorid und dem Cholesten findet eine Addition unter Bildung der entsprechenden Cholestankörper: Chlorcholestanol, Dichlorcholestan und Chlorcholestan statt. Mit diesen Körpern beschäftigt sich die folgende Mitteilung.

\section{Chlorcholestanol (Cholesterinchlorhydrat).}

Die Darstellung dieser Verbindung geschah in folgender Weise: Je $20 \mathrm{~g}$ entwässertes Cholesterin wurden in $300 \mathrm{~cm}^{3}$ Äther gelöst und diese Lösung mit $300 \mathrm{~cm}^{3}$ absoluten Alkohols versetzt; hierauf wurde unter Wasserkühlung (im Sommer unter Eiskühlung) ein rascher Strom von trockenem Chlorwasserstoff eingeleitet. $\mathrm{Da}$ schon während des Einleitens eine reichliche Ausscheidung von kristallinischen Massen eintritt, muß dafür gesorgt werden, daß eine Verstopfung des $\mathrm{Zu}-$ leitungsrohres behoben werden kann. Das Einleiten des Gases wurde so lange fortgesetzt, bis die Gewichtszunahme zirka $200 \mathrm{~g}$ betrug, was gewöhnlich einen Tag in Anspruch nahm. Nach einigen Stunden erstarrt die Masse zu einem Filz feiner, farbloser Nadeln, die eingeschlossene Flüssigkeit nimmt eine rötliche, später braunrote Farbe an. Nach ein- bis zweitägigem Stehen wurden die Kristalle durch Absaugen von der Lösung getrennt und unter einer Glocke über konzentrierter Schwefelsäure und Ätzkali getrocknet. Je $20 \mathrm{~g}$ Cholesterin lieferten so 10 bis $12 \mathrm{~g}$ des rohen Additionsproduktes, das jedoch noch weiterer Reinigung bedarf; bei zahlreichen Darstellungen ergab sich ein Chlorgehalt von 9 bis $9.5 \%$, was darauf hinweist, daß neben dem einfachen Anlagerungsprodukt, das nach der Rechnung $8 \cdot 42 \%$ Chlor enthält, noch eine chlorreichere Verbindung gebildet worden sein muß. Eine solche wurde später auch in der Tat nachgewiesen. Läßt man den Kristallbrei längere Zeit in der Flüssigkeit stehen, so geht er allmählich ganz in Lösung und es scheiden sich - gerade so wie beim längeren Stehen der abgesaugten Mutterlauge - Kristalle von $\beta$-Dichlorcholestan aus. 
In rein ätherischer Lösung läßt sich die Anlagerung nicht erzielen; schon nach den ersten Blasen von Chlorwasserstoff scheiden sich so reichliche Mengen von Kristallnadeln aus, daß der Gasstrom unterbrochen werden muß; das Ausgeschiedene ist nichts anderes als wasserfreies Cholesterin.

In Chloroform geht die Reaktion vor sich, doch scheint es, daß dem Produkt größere Mengen des $\beta$-Dichlorcholestans beigemengt sind.

Die Reinigung des Chlorcholestanols geschah entweder durch Lösen in trockenem Äther und Fällen mit Alkohol oder durch Versetzen einer gesättigten Lösung in Chloroform mit dem drei- bis vierfachen Volumen Petroleumäther. In beiden Fällen scheiden sich äußerst feine, seidenglänzende, biegsame Nadeln aus, die beim Absaugen auf ein kleines Volumen zusammenschrumpfen. Bei der Anwendung heißer Lösungsmittel tritt leicht Zersetzung ein; wasserhaltiger Alkohol liefert dabei nach dem Erkalten eine durchscheinende, gelatinöse Masse.

Das Chlorcholestanol löst sich in Chloroform, Äther, Benzol, Schwefelkohlenstoff ziemlich leicht, schwerer in Alkohol. Im Kapillarröhrchen erhitzt, beginnt es gegen $150^{\circ}$ stark zu schwinden, bei 154 bis $155^{\circ}$ schmilat es unter Gasentwicklung. Erhitzt man eine Probe in der Eprouvette, so destilliert unter Entweichen von Salzsäure ein Öl, das allmählich kristallinisch erstarrt, möglicherweise ein Cholesterilen.

Die Analyse gab folgende Zahlen:

I. $0.2562 \mathrm{~g}$, im Vakuum über Schwefelsäure getrocknet, gaben $0.2533 \mathrm{~g}$ Wasser und $0.7292 \mathrm{~g}$ Kohlensäure.

II. $0.4175 \mathrm{~g}$, mit Natriummethylat durch zwei Stunden gekocht, verbrauchten $9 \cdot 7 \mathrm{~cm}^{3} n / 10$ Silberlösung.

III. $0 \cdot 4819 \mathrm{~g}$ verbrauchten $11 \cdot \mathrm{g} n / 10$ Silberlösung.

In 100 Teilen:
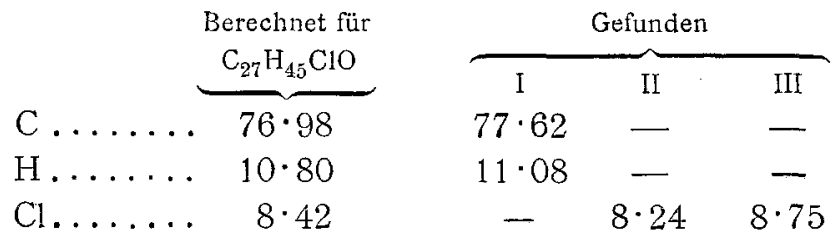
Daß es sich in der vorliegenden Verbindung tatsächlich um ein Produkt der Anlagerung von Chlorwasserstoff an die doppelte Bindung handelt, ergibt sich daraus, daßs sie nicht mehr Brom zu addieren vermag.

$0 \cdot 1 \mathrm{~g}$ in $5 \mathrm{~cm}^{3}$ Chloroform gelöst, wurden mit einer Lösung von Brom in Chloroform 1: 10 versetzt; schon nach dem Zusatz von $0.05 \mathrm{~cm}^{3}$ blieb die Farbe des Broms bestehen. In unserer ersten Abhandlung über Cholesterin haben Suida und ich über Unterschiede berichtet, die bei den Cholesten- und den Cholestankörpern in ihrem Verhalten gegen einige Reagenzien bestehen. ${ }^{1}$ Die Cholestankörper sind viel weniger reaktionsfähig gegenüber konzentrierter Schwefelsäure, zeigen bei der Probe mit Chloroform und Schwefelsäure keine oder nur ganz schwache Reaktion und werden durch rauchende Salpetersäure nicht angegriffen. Das Chlorcholestanol zeigt diese Widerstandsfähigkeit gegenüber konzentrierter Schwefelsäure nicht; es wird Salzsäure abgespalten und daher tritt die Reaktion wie beim Cholesterin ein, ebenso bei der Probe mit Chloroform und Schwefelsäure. Dagegen erweist sich das Chlorcholestanol im Verhalten gegen rauchende Salpetersäure als echter Cholestankörper: es tritt keine Verflüssigung und keine Reaktion ein, wenn man es bei gewöhnlicher Temperatur damit stehen läßt.

Es wurde bereits mitgeteilt, ${ }^{2}$ daß aus dem Produkt der Anlagerung von Chlorwasserstoff an Cholesterin ein Körper von der Zusammensetzung des Ausgangsmaterials zurückgewonnen werden kann; die Identität mit dem gewöhnlichen Cholesterin mußte uns anfangs fraglich erscheinen, da das optische Verhalten des Abspaltungsproduktes von dem des Cholesterins abwich. Es war denkbar, daß es auf diesem Wege zur Entstehung eines is omeren Cholesterins kommen könnte, weshalb die Reaktion genauer verfolgt werden mußte. ${ }^{3}$ Dabei ergab sich nun, daß die Abspaltung von Chlorwasserstoff nicht

1 Monatshefte für Chemie, Bd. XV (1894), p. 112.

2 Mauthner und Suida, Monatshefte für Chemie, Bd. XXIV (1903), p. 664 .

3 Ein Bindungswechsel, so wie er bei der Einwirkung von Chlorwasserstoff auf Pinen eintritt, schien nicht ausgeschlossen. 
ganz glatt verläuft; es gelingt wohl, das gewöhnliche Cholesterin aus dem Reaktionsprodukt $z u$ isolieren; es ist aber mit einem Öl gemengt und kann erst durch oftmaliges Umkristallisieren, am besten nach Überführung in das essigsaure Salz, rein erhalten werden. Es ist also nicht ausgeschlossen, daß neben dem ursprünglichen Cholesterin bei der Reaktion ein Isomeres gebildet wird, dessen Isolierung jedoch nicht möglich war. Auf die schwierige Reinigung ist auch die seinerzeit beobachtete Differenz im Drehungsvermögen zurückzuführen.

Die Abspaltung geschah in alkoholischer Lösung mit Kaliumhydroxyd oder Kaliumacetat; nach der Reinigung gab das wiedergewonnene Cholesterin, das den Schmelzpunkt von $147^{\circ}$ zeigte, bei der Analyse folgende Zahlen:

I. $0.2669 \mathrm{~g}$ gaben bei $100^{\circ} 0.011 \mathrm{~g}$ Wasser.

II. $0 \cdot 237 \mathrm{~g}$ gaben $0 \cdot 0111 \mathrm{~g}$ Wasser.

In 100 Teilen:

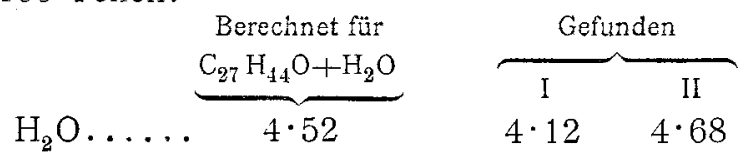

III. $0 \cdot 2559 \mathrm{~g}$ gaben nach dem Trocknen $0 \cdot 2672 \mathrm{~g}$ Wasser und $0 \cdot 7899 \mathrm{~g}$ Kohlensäure.

IV. $0.2287 \mathrm{~g}$ gaben $0.2463 \mathrm{~g}$ Wasser und $0 \cdot 7034 \mathrm{~g}$ Kohlensäure.

In 100 Teilen:

\begin{tabular}{|c|c|c|c|}
\hline & \multirow{2}{*}{$\begin{array}{r}\begin{array}{c}\text { Berechnet für } \\
\mathrm{C}_{27} \mathrm{H}_{44} \mathrm{O}\end{array} \\
\end{array}$} & \multicolumn{2}{|c|}{ Gefunden } \\
\hline & & III & IV \\
\hline & $84 \cdot 28$ & $84 \cdot 18$ & $83 \cdot 88$ \\
\hline $\mathrm{H}$ & $11 \cdot 56$ & $11 \cdot 70$ & $12 \cdot 07$ \\
\hline
\end{tabular}

Bei der Bestimmung des Drehungsvermögens in Äther ergaben sich folgende Zahlen:

$$
\begin{aligned}
& c=3 \cdot 1472 \\
& l=2 \\
& \alpha=-1 \cdot 87^{\circ} \\
& {[\alpha]_{D}=-29 \cdot 7^{\circ}}
\end{aligned}
$$


Dies steht mit der Bestimmung von R. Burian ${ }^{1}\left(-29 \cdot 9^{\circ}\right)$ in genügender Übereinstimmung.

Die Vermutung, es könnte neben dem gewöhnlichen Cholesterin ein Isomeres von anderem Drehungsvermögen entstehen, findet eine Stütze in der Beobachtung, daß bei der fortschreitenden Reinigung durch wiederholtes Umkristallisieren viel früher die quantitativ richtige Zusammensetzung erreicht wird als der richtige Schmelzpunkt und das richtige Drehungsvermögen.

\section{2. $\beta$-Dichlorcholestan.}

Wie früher erwähnt wurde, scheidet sich aus der vom Chlorcholestanol abfiltrierten Lösung bei längerem Stehen eine neue Substanz aus. Es sind weiße, kristallinische Körner, die im Laufe von 3 bis 4 Wochen an Menge zunehmen; aus je $20 \mathrm{~g}$ Cholesterin wurden so 4 bis $4.5 \mathrm{~g}$ erhalten. Die Kristallkörner wurden von der inzwischen ganz dunkel gefärbten Flüssigkeit abfiltriert, mit Alkohol gewaschen und mehrmals aus einem Gemenge von 1 Volum Benzol und 9 Volumen Alkohol umkristallisiert, wobei flache Nadeln vom Schmelzpunkt $117^{\circ}$ resultierten. Ihre Analyse ergab, daß ein sauerstofffreier chlorhaltiger Körper von der Zusammensetzung $\mathrm{C}_{27} \mathrm{H}_{44} \mathrm{Cl}_{2}$ vorliegt.

I. $0 \cdot 1432 \mathrm{~g}$ gaben $0 \cdot 1373 \mathrm{~g}$ Wasser und $0.3877 \mathrm{~g}$ Kohlensäure.

II. $0.2114 \mathrm{~g}$ gaben $0.2017 \mathrm{~g}$ Wasser und $0.5708 \mathrm{~g}$ Kohlensäure.

III. $0.3006 \mathrm{~g}$ gaben $0.19 \mathrm{~g}$ Chlorsilber und $0.0025 \mathrm{~g}$ Silber.

IV. $0 \cdot 3066 \mathrm{~g}$ gaben $0 \cdot 1925 \mathrm{~g}$ Chlorsilber und $0.0082 \mathrm{~g}$ Silber. In 100 Teilen:

\begin{tabular}{|c|c|c|c|c|c|}
\hline & Berechnet für & & Geft & den & \\
\hline & $\mathrm{C}_{27} \mathrm{H}_{44} \mathrm{Cl}_{2}$ & I & II & III & IV \\
\hline C & $73 \cdot 75$ & $73 \cdot 84$ & $73 \cdot 64$ & - & - \\
\hline & $10 \cdot 11$ & $10 \cdot 75$ & $10 \cdot 70$ & - & - \\
\hline $\mathrm{Cl} \ldots \ldots \ldots$ & $16 \cdot 14$ & $\ldots$ & - & $15 \cdot 90$ & $16 \cdot 40$ \\
\hline
\end{tabular}

1 Monatshefte für Chemie, Bd. XVIII (1897), p. 555. 
Die Bestimmung des Molekulargewichtes in Naphtalin ergab folgendes:

$\begin{array}{ccccc}\text { Substanz } & \text { Naphtalin } & \text { Erniedrigung } & \overbrace{\text { Gefunden }}^{\text {Molekulargewicht }} & \text { Berechnet } \\ 0 \cdot 1929 & 15 & 0 \cdot 215^{\circ} & 413 & 439 \cdot 34 \\ 0 \cdot 3198 & 15 & 0 \cdot 350^{\circ} & 420 & \end{array}$

Wie die Analyse zeigt, ist die Substanz durch weitere Einwirkung des Chlorwasserstoffes aus Chlorcholestanol entstanden, wobei die Hydroxylgruppe durch Chlor ersetzt wurde, ein Vorgang, der sich durch die Gleichungen ausdrücken läßt:

$$
\begin{aligned}
& \text { I. } \mathrm{C}_{27} \mathrm{H}_{43} \mathrm{OH}+\mathrm{HCl}=\mathrm{C}_{27} \mathrm{H}_{44} \mathrm{ClOH} \\
& \text { II. } \mathrm{C}_{27} \mathrm{H}_{44} \mathrm{ClOH}+\mathrm{HCl}=\mathrm{C}_{27} \mathrm{H}_{44} \mathrm{Cl}_{2}+\mathrm{H}_{2} \mathrm{O} \text {. }
\end{aligned}
$$

Ein Körper von gleicher Zusammensetzung ist bereits vor langer Zeit beschrieben worden; es ist das Cholestendichlorid, das durch Anlagerung von Chlor an Cholesten $\mathrm{C}_{27} \mathrm{H}_{44}$ gewonnen wurde. ${ }^{1}$ Durch Aussehen, Schmelzpunkt und optisches Drehungsvermögen unterscheiden sich die beiden Körper wesentlich voneinander. Zum Unterschied von dem Cholestendichlorid ( $\alpha$-Dichlorcholestan) soll der neue isomere Körper $\beta$-Dichlorcholestan genannt werden.

Über die Bindung der beiden Chloratome läßt sich so viel aussagen, daß eines von ihnen in beiden Isomeren die gleiche

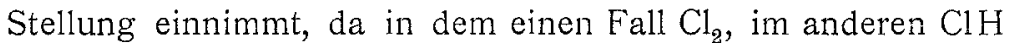
an die Doppelbindung angelagert wurde, während das zweite in den beiden Isomeren verschieden gelagert sein muß: beim Cholestendichlorid dem ersteren sicher benachbart, beim $\beta$-Dichlorcholestan an Stelle der Hydroxylgruppe des Cholesterins, die im Cholesten durch Wasserstoff vertreten ist.

Es lag nahe, zu versuchen, ob man das $\beta$-Dichlorcholestan auch aus dem Cholesterylchorid durch Anlagerung

1 Mauthner und Suida, I. Abhandlung. Monatshefte für Chemie, Bd. XV (1894), p. 95. 
von Chlorwasserstoff gewinnen kann. Es ist in der Tat der Fall; dieser Weg der Darstellung ist sogar der bequemere.

$5 \mathrm{~g}$ Cholesterylchlorid wurden in $100 \mathrm{~cm}^{3}$ trockenem Äther gelöst, die Lösung mit $100 \mathrm{~cm}^{3}$ Alkohol versetzt und mit Salzsäuregas behandelt. Nach einigen Stunden fanden sich reichlich feine Nadeln ausgeschieden, daneben aber auch Blättchen offenbar noch unverändertes Chlorid. Nach drei Tagen waren diese Blättchen verschwunden und nur nadelförmige Kristalle erkennbar. Abgesaugt, mit Alkohol gewaschen und an der Luft getrocknet, besaßen sie das Gewicht von $4 \cdot 8 \mathrm{~g}$. Durch siebenmaliges Umkristallisieren aus Benzol-Alkohol (1:9) wurden sie rein erhalten; sie zeigten dann den Schmelzpunkt $117^{\circ}$, das gleiche Aussehen und (bis auf eine kleine, innerhalb der Versuchsfehler fallende Differenz) das gleiche Drehungsvermögen wie die vorhin beschriebenen Kristalle von $\beta$-Dichlorcholestan; sie lieferten bei der Analyse folgende Zahlen:

I. $0 \cdot 2052 \mathrm{~g}$ gaben $0 \cdot 1943 \mathrm{~g}$ Wasser und $0.552 \mathrm{~g}$ Kohlensäure.

II. $0.2892 \mathrm{~g}$ gaben $0 \cdot 1778 \mathrm{~g}$ Chlorsilber und $0.0064 \mathrm{~g}$ Silber.

In 100 Teilen :

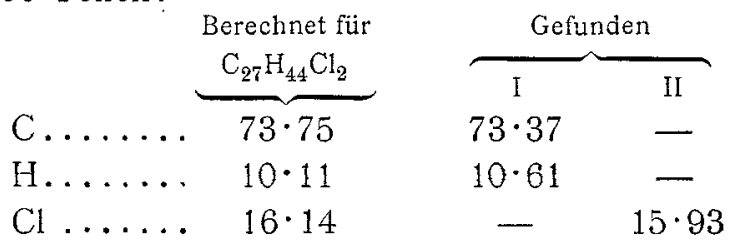

Das $\beta$-Dichlorcholestan kann somit als das Chlorhydrat des Cholesterylchlorids aufgefaßt werden.

In Chloroformlösung fand eine Anlagerung von Chlorwasserstoff an Cholesterylchlorid nicht statt; dieses wurde unverändert zurückerhalten.

Das $\beta$-Dichlorcholestan ist in Äther, Benzol, Chloroform, Schwefelkohlenstoff leicht, in Alkohol schwerer löslich. Es addiert kein Brom; trocken erhitzt, liefert es Salzsäure und ein kristallinisch erstarrendes Destillat. Seine Reaktionen entsprechen seiner Natur als Cholestankörper: von Schwefelsäure wird es in der Kälte nicht sofort angegriffen; erst bei längerer 
Berührung damit färbt sich die Säure ganz wenig und nimmt dabei schwachgrüne Fluoreszenz an. Die SchwefelsäureChloroformreaktion fällt negativ aus; von rauchender Salpetersäure wird es nicht angegriffen.

\section{Chlorcholestan (Cholestylchlorid).}

$3 \mathrm{~g}$ reines, bei $91^{\circ}$ schmelzendes Cholesten wurde in $50 \mathrm{~cm}^{3}$ Chloroform gelöst und mit Salzsäuregas behandelt; nach zwei Tagen wurde die rosafarbige, gelb fluoreszierende Lösung verdunstet, der Rückstand erstarrte allmählich vollständig kristallinisch. Durch Auflösen in Äther und Versetzen mit Alkohol wurde die Substanz umkristallisiert; es schieden sich zunächst prächtige glasglänzende, flache Prismen aus, die zum Teil eine Länge von 1 bis $2 \mathrm{~cm}$ erreichten; später kamen feine irisierende Blättchen dazu, die meist zu Büscheln vereinigt sind.

Die Prismen wurden isoliert, mit Alkohol abgespült und im Vakuum getrocknet. Beim Erhitzen beginnen sie bei etwa $80^{\circ} \mathrm{zu}$ sintern und sind bei $91^{\circ}$ ganz geschmolzen; nach dem Erkalten wird die geschmolzene Masse wieder kristallinisch. Die Analyse ergab folgende Zahlen:

I. $0 \cdot 1856 \mathrm{~g}$ gaben $0 \cdot 1930 \mathrm{~g}$ Wasser und $0.541 \mathrm{~g}$ Kohlensäure;

II. $0.315 \mathrm{~g}$ gaben $0.0992 \mathrm{~g}$ Chlorsilber und $0.0082 \mathrm{~g}$ Silber. III. $0.2192 \mathrm{~g}$ gaben $0.0697 \mathrm{~g}$ Chlorsilber und $0.007 \mathrm{~g}$ Silber. In 100 Teilen:

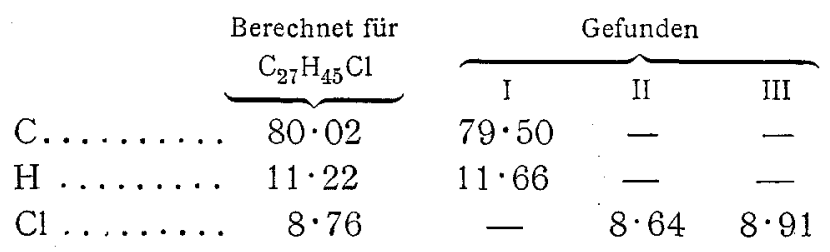

Die Löslichkeitsverhältnisse sind beim Chlorcholestan ähnlich wie beim $\beta$-Dichlorcholestan; in siedendem Alkohol schmilzt es zu einem farblosen Ö1. In Chloroform addiert es kein Brom. 
Es muß weiteren Versuchen vorbehalten bleiben, wie sich die beschriebenen Verbindungen in Bezug auf die Reaktionsfähigkeit des in ihnen enthaltenen Chlors verhalten und ob es gelingt, dieses letztere durch andere Gruppen zu ersetzen. Auch Versuche über die Anlagerung von Bromwasserstoff sollen in Angriff genommen werden.

Über das optische Verhalten der beschriebenen Körper soll im Zusammenhang mit den Ergebnissen der Drehungsbestimmung an anderen Cholestankörpern in nächster Zeit berichtet werden. Es sei hier nur mitgeteilt, daß die Linksdrehung, welche Cholesten, Cholesterin und Cholesterylchlorid zeigen, durch die Anlagerung von Chlorwasserstoff in Rechtsdrehung übergeht. 